\title{
Estimation of Nonlinear Dynamic Panel Data Models with Individual Effects
}

\author{
Yi Hu, ${ }^{1}$ Dongmei Guo, ${ }^{2}$ Ying Deng, ${ }^{3}$ and Shouyang Wang ${ }^{4}$ \\ ${ }^{1}$ School of Management, University of Chinese Academy of Sciences, Beijing 100190, China \\ ${ }^{2}$ School of Economics, Central University of Finance and Economics, Beijing 100081, China \\ ${ }^{3}$ School of International Trade and Economics, University of International Business and Economics, Beijing 100029, China \\ ${ }^{4}$ Academy of Mathematics and Systems Science, Chinese Academy of Sciences, Beijing 100190, China \\ Correspondence should be addressed to Dongmei Guo; guodongmeicufe@163.com
}

Received 6 December 2013; Accepted 22 January 2014; Published 25 February 2014

Academic Editor: Chuangxia Huang

Copyright (c) $2014 \mathrm{Yi} \mathrm{Hu}$ et al. This is an open access article distributed under the Creative Commons Attribution License, which permits unrestricted use, distribution, and reproduction in any medium, provided the original work is properly cited.

\begin{abstract}
This paper suggests a generalized method of moments (GMM) based estimation for dynamic panel data models with individual specific fixed effects and threshold effects simultaneously. We extend Hansen's (Hansen, 1999) original setup to models including endogenous regressors, specifically, lagged dependent variables. To address the problem of endogeneity of these nonlinear dynamic panel data models, we prove that the orthogonality conditions proposed by Arellano and Bond (1991) are valid. The threshold and slope parameters are estimated by GMM, and asymptotic distribution of the slope parameters is derived. Finite sample performance of the estimation is investigated through Monte Carlo simulations. It shows that the threshold and slope parameter can be estimated accurately and also the finite sample distribution of slope parameters is well approximated by the asymptotic distribution.
\end{abstract}

\section{Introduction}

Since many economic relationships are dynamic and nonlinear, nonlinear/dynamic panel data models could obtain more information from data sources than traditional models $[1,2]$. For example, many researchers suggest that economic growth is a nonlinear process [3-5] and a number of empirical analyses of economic growth entail dynamic econometric models [6-9], with lagged dependent variable among the regressors. However, few researchers consider the dynamic and nonlinear relationships simultaneously and the purpose of this paper is to combine these two factors in one model.

Many results exist in the theoretical literature concerning the estimation and inference for dynamic panel data models. Since the lagged dependent variables and the disturbance term are correlated due to the unobserved effects, standard least square methods could not obtain consistent estimators when the model is dynamic. To overcome this problem, Anderson and Hsiao [6] suggested that we difference the model first to get rid of the unobserved effects and then use instrumental variable (IV) estimation for the transformed model. Nevertheless, this IV estimation method leads to consistent but not necessarily efficient estimates of the parameters because it does not use all the available moment conditions. Arellano and Bond [10] proposed a generalized method of moments (GMM) procedure that is more efficient than the Anderson and Hsiao [6] estimator. This literature is generalized and extended by Arellano and Bover [11] and Blundell and Bond [12], which are called forward orthogonal deviation and system GMM, respectively. For the latest development of dynamic panel data models, see Baltagi [13] and Han and Phillips [14] for more details.

Several models could be chosen to describe the nonlinear relationship such as mixture models, switching models, smooth transition threshold models, and threshold models. In this paper threshold model is used because of wide applications in empirical researches. This model splits the sample into classes based on an observed variable - whether or not it exceeds some thresholds. In most situations, the complexity of the problem increases because the exact threshold is unknown and needed to be estimated. The estimation and inference are fairly well developed for linear models with 
exogenous regressors [15-17], in which only the nondynamic case is considered.

The dynamic panel threshold models have been used in empirical literature. Cheng et al. [18] examined the evidence on the conditional convergence growth theory, which extended dynamic panel data growth model to control both threshold effects and cross-section dependence. Chong et al. [19] studied the relationship between the depletion rate of foreign reserves and currency crises using threshold autoregressive model. Ho [20] applied a dynamic panel threshold model to examine whether the low-income countries catch up with the rich ones. Kremer et al. [21] considered a dynamic panel threshold model to study inflation thresholds for longterm economic growth. As Hansen's model required that all regressors are exogenous, the method of Hansen [17] used in these papers to estimate the dynamic models may not be suitable due to the lagged dependent variables. So far, the theory of dynamic panel threshold model has not been available as we know except for Dang et al. [22]. However, the validity of the instrumental matrices is not proved. This paper proposes an estimation method for dynamic panel threshold model and our analysis mainly relies on Hansen [17], Arellano and Bond [10], and Caner and Hansen [16]. First, we prove that the orthogonality conditions considered in ordinary dynamic panel data models are also valid in nonlinear dynamic models. Second, we develop a GMM estimator of the threshold and slope parameters based on the above moment conditions.

The remainder of the paper proceeds as follows. Section 2 introduces the model and notations. Section 3 discusses the estimation for the threshold and slope coefficients. Section 4 reports a Monte Carlo simulation, and Section 5 concludes.

\section{Model}

Consider a simple AR(1) model without exogenous variables but with individual and threshold effects as shown in the following structural equation:

$$
\begin{gathered}
y_{i t}=\mu_{i}+\alpha_{1} y_{i t-1} I\left(q_{i t} \leq \gamma\right)+\alpha_{2} y_{i t-1} I\left(q_{i t}>\gamma\right)+\varepsilon_{i t}, \\
\text { for } i=1, \ldots, N ; \quad t=1, \ldots, T
\end{gathered}
$$

where $i$ denotes cross-sections and $t$ denotes time. $y_{i t}$ denotes the observable dependent variable; $q_{i t}$ denotes the exogenous threshold variable; $\gamma$ denotes the threshold parameter, which is assumed to be unknown and needs to be estimated; $\alpha_{j}$ denotes a parameter that satisfies $\left|\alpha_{j}\right|<1(j=1,2) ; \mu_{i}$ is the unobserved individual effect; $\varepsilon_{i t}$ is the idiosyncratic error, which is assumed to be independent and identically distributed (i.i.d) with mean zero and variance $\sigma^{2}$ conditional on $\mu_{i}, y_{i, t-1}, \ldots, y_{i 0} . I(\cdot)$ is indicator function.

One can also write (1) in the form

$$
\begin{array}{ll}
y_{i t}=\mu_{i}+\alpha_{1} y_{i t-1}+\varepsilon_{i t}, & q_{i t} \leq \gamma, \\
y_{i t}=\mu_{i}+\alpha_{2} y_{i t-1}+\varepsilon_{i t}, & q_{i t}>\gamma .
\end{array}
$$

For simplicity, we assume that $y_{i 0}$ is observed and let $x_{i t} \equiv$ $y_{i, t-1}$. Alternatively, (1) can also be written compactly as

$$
y_{i t}=\mu_{i}+x_{i t}(\gamma)^{\prime} \alpha+\varepsilon_{i t},
$$

where $x_{i t}(\gamma)=\left(x_{i t} I\left(q_{i t} \leq \gamma\right), x_{i t} I\left(q_{i t}>\gamma\right)\right)^{\prime}, \alpha=\left(\alpha_{1}, \alpha_{2}\right)^{\prime}$.

\section{Estimation}

In this section, we first consider a simple model without exogenous covariates and derive GMM based estimator for the threshold parameter $\gamma$ and slope parameters $\alpha$. Then we extend the simple model to cases with strictly exogenous covariates.

3.1. Estimation of Threshold and Slope Parameters. In traditional dynamic panel data model, two methods are commonly used to remove individual effect $\mu_{i}$. One is firstdifference approach suggested by Arellano and Bond [10]; the other one is forward orthogonal deviation proposed by Arellano and Bover [11]. We will utilize the first-difference approach in the following derivation, due to the fact that it is more convenient for computation.

First, we take first-difference for model (3) to get rid of the time invariant individual effects

$$
\Delta y_{i t}=\Delta x_{i t}(\gamma)^{\prime} \alpha+\Delta \varepsilon_{i t},
$$

where $\Delta$ denotes difference operator. If $\alpha_{1}=\alpha_{2}$, that is, there is no threshold effect, then additional instruments can be obtained in dynamic panel data models if one utilizes the orthogonality conditions that exist between lagged values of $y_{i t}$ and the disturbance $\varepsilon_{i t}$ according to Arellano and Bond [10]. Here we prove that these orthogonality conditions are also valid in model (4) when $\alpha_{1} \neq \alpha_{2}$.

For any given $t$, we have either $x_{i t}(\gamma)=\left(x_{i t}, 0\right)^{\prime}$ or $x_{i t}(\gamma)=$ $\left(0, x_{i t}\right)^{\prime}$. Consider the former one without loss of generality. Similarly, there must be two cases in the period $t-1$,

$$
\begin{aligned}
& \text { Case 1: } x_{i, t-1}(\gamma)=\left(x_{i, t-1}, 0\right)^{\prime} . \\
& \text { Case 2: } x_{i, t-1}(\gamma)=\left(0, x_{i, t-1}\right)^{\prime} .
\end{aligned}
$$

Then first difference yields

$$
\Delta x_{i t}(\gamma)= \begin{cases}\left(\Delta x_{i, t}, 0\right)^{\prime}, & \text { for case } 1, \\ \left(x_{i, t},-x_{i, t-1}\right)^{\prime}, & \text { for case } 2 .\end{cases}
$$

Correspondingly,

$$
\Delta x_{i t}(\gamma)^{\prime} \alpha= \begin{cases}\alpha_{1} \Delta x_{i, t}=\alpha_{1} x_{i, t}-\alpha_{1} x_{i, t-1}, & \text { for case } 1 \\ \alpha_{1} x_{i, t},-\alpha_{2} x_{i, t-1} & \text { for case } 2\end{cases}
$$

For any given $t, x_{i, t-1}$ is a valid instrument (A valid instrument means that it should have two basic conditions: first, erogeneity, i.e., it should be independent of (or, at least, uncorrelated with) the disturbance term in the equation of interest; second, relevance, i.e., it should be correlated with the included endogenous explanatory variables for which it 
is supposed to serve as an instrument.) for both case 1 and case 2 since it is correlated with $\Delta x_{i t}(\gamma)^{\prime} \alpha$, that is, $\alpha_{1} x_{i, t}-$ $\alpha_{1} x_{i, t-1}$ or $\alpha_{1} x_{i, t},-\alpha_{2} x_{i, t-1}$, but not correlated with $\Delta \varepsilon_{i t}$, that is, $\varepsilon_{i t}-\varepsilon_{i, t-1}$ as long as $\varepsilon_{i t}$ 's are serially uncorrelated. Given the autoregressive nature of the model and the assumption that there is no serial correlation in $\varepsilon_{i t}$, it can be easily shown that $x_{i, 1}, \ldots, x_{i, t-2}$ are also valid instruments. Therefore, the orthogonality conditions are given by

$$
E\left(x_{i, t-s} \Delta \varepsilon_{i t}\right)=0, \quad \text { for } s=1, \ldots, t-1 ; t=2, \ldots, T \text {. }
$$

Define

$$
Z_{i}=\left(\begin{array}{cccc}
x_{i 1} & 0 & \cdots & 0 \\
0 & x_{i, 1}, x_{i, 2} & \cdots & 0 \\
0 & 0 & \cdots & 0 \\
\vdots & \vdots & \ddots & \vdots \\
0 & 0 & \cdots & x_{i, 1}, x_{i, 2}, \ldots x_{i, T-1}
\end{array}\right)
$$

then for each $i$ the $T(T-1) / 2$ moment conditions described above can be written as

$$
E\left(Z_{i}^{\prime} \Delta \varepsilon_{i}\right)=0
$$

Note that $\Delta \varepsilon_{i t}=\varepsilon_{i t}-\varepsilon_{i, t-1}$ is $\operatorname{MA}(1)$. Define $\Delta \varepsilon_{i}=$ $\left(\Delta \varepsilon_{i 2}, \cdots, \Delta \varepsilon_{i T}\right)^{\prime}$; then

$$
E\left(\Delta \varepsilon_{i} \Delta \varepsilon_{i}^{\prime}\right)=\sigma^{2} G
$$

where

$$
G=\left(\begin{array}{cccccc}
2 & -1 & 0 & \cdots & 0 & 0 \\
-1 & 2 & -1 & \cdots & 0 & 0 \\
\vdots & \vdots & \vdots & \ddots & \vdots & \vdots \\
0 & 0 & 0 & \cdots & 2 & -1 \\
0 & 0 & 0 & \cdots & -1 & 2
\end{array}\right)
$$

Let $\widehat{\alpha}_{\mathrm{GMM}}(\gamma)$ be the GMM estimator with moment conditions given by (10). The GMM estimator can be written as

$$
\begin{aligned}
\widehat{\alpha}_{\mathrm{GMM}}(\gamma) & \left\{\left[\sum_{i=1}^{N} \Delta X_{i}(\gamma)^{\prime} Z_{i}\right]\left[\sum_{i=1}^{N} Z_{i}^{\prime} G Z_{i}\right]^{-1}\left[\sum_{i=1}^{N} Z_{i}^{\prime} \Delta X_{i}(\gamma)\right]\right\}^{-1} \\
& \cdot\left\{\left[\sum_{i=1}^{N} \Delta X_{i}(\gamma)^{\prime} Z_{i}\right]\left[\sum_{i=1}^{N} Z_{i}^{\prime} G Z_{i}\right]^{-1}\left[\sum_{i=1}^{N} Z_{i}^{\prime} \Delta Y_{i}\right]\right\}
\end{aligned}
$$

where $\Delta X_{i}(\gamma)=\left[\Delta x_{i 2}(\gamma), \ldots, \Delta x_{i T}(\gamma)\right]^{\prime}, \Delta Y_{i}=$ $\left(\Delta y_{i 2}, \ldots, \Delta y_{i T}\right)^{\prime}$.

Stacking over individuals, (13) can be written compactly as

$$
\begin{aligned}
\widehat{\alpha}_{\mathrm{GMM}}(\gamma)= & {\left[\Delta X(\gamma)^{\prime} Z\left(Z^{\prime} \Omega Z\right)^{-1} Z^{\prime} \Delta X(\gamma)\right]^{-1} } \\
& \times\left[\Delta X(\gamma)^{\prime} Z\left(Z^{\prime} \Omega Z\right)^{-1} Z^{\prime} \Delta Y\right],
\end{aligned}
$$

where $\Omega=I_{N} \otimes G, \otimes$ is Kronecker product and $\Delta X(\gamma)=$ $\left[\Delta X_{1}(\gamma)^{\prime}, \ldots, \Delta X_{N}(\gamma)^{\prime}\right]^{\prime}, \Delta Y=\left[\Delta Y_{1}^{\prime}, \ldots, \Delta Y_{N}^{\prime}\right]^{\prime}$, and $Z=$ $\left(Z_{1}^{\prime}, \ldots, Z_{N}^{\prime}\right)^{\prime}$.

In fact, this estimator is infeasible in empirical studies, since it depends on an unknown parameter $\gamma$. Therefore, our next step is to estimate $\gamma$ from the regression residuals:

$$
\widehat{e}(\gamma)=\Delta Y-\Delta X(\gamma) \widehat{\alpha}_{\mathrm{GMM}}(\gamma) .
$$

We apply the estimator suggested by Chan [23] and Hansen $[15,17]$; then $\gamma$ can be estimated by

$$
\widehat{\gamma}=\underset{\gamma}{\operatorname{argmin}} S(\gamma)
$$

where $S(\gamma)=\widehat{e}(\gamma)^{\prime} \widehat{e}(\gamma)$ is the sum of squared errors.

Once $\hat{\gamma}$ is obtained, we substitute the true parameter $\gamma$ with its estimate $\hat{\gamma}$ yielding the feasible GMM estimator of slope coefficient estimate:

$$
\widehat{\alpha}=\widehat{\alpha}_{\mathrm{GMM}}(\widehat{\gamma}) \text {. }
$$

According to Hansen [24], under the case of known $\gamma$, GMM estimator $\widehat{\alpha}_{\mathrm{GMM}}(\gamma)$ is efficient and asymptotically normal:

$$
\sqrt{N}\left(\widehat{\alpha}_{\mathrm{GMM}}(\gamma)-\alpha\right) \Longrightarrow N(0, V) \text { as } N \longrightarrow \infty,
$$

where

$$
V=N\left[\Delta X(\gamma)^{\prime} Z\left(\sigma^{2} G\right)^{-1} Z^{\prime} \Delta X(\gamma)\right]^{-1}
$$

Hansen [17] and Caner and Hansen [16] show that the dependence on the threshold estimate is not of first-order asymptotic importance, so inference on $\widehat{\alpha}$ could proceed as if the estimated threshold parameter $\widehat{\gamma}$ was the true parameter $\gamma$. Then,

$$
\sqrt{N}(\widehat{\alpha}-\alpha) \Longrightarrow N(0, V) \text { as } N \longrightarrow \infty .
$$

The estimated covariance matrix becomes (One can also consider Windmeijer's bias-corrected estimator (Windmeijer [25]) for the robust VCE of two-step GMM estimators.)

$$
\widehat{V}=N\left[\Delta X(\widehat{\gamma})^{\prime} Z \widehat{G}^{-1} Z^{\prime} \Delta X(\widehat{\gamma})\right]^{-1}
$$

where $\widehat{G}=\sum_{i=1}^{N} Z_{i}^{\prime}\left(\widehat{e}_{1}(\widehat{\gamma}) \widehat{e}_{1}(\widehat{\gamma})^{\prime}\right) Z_{i}$ and $\widehat{e}_{1}(\widehat{\gamma})=\Delta Y-\Delta X(\widehat{\gamma}) \widehat{\alpha}$. Similarly, one can prove that $\widehat{V}$ converges in probability to $V$ as in Caner and Hansen [16].

3.2. Estimation of the Model with Exogenous Variables. Now we extend the results in the previous subsection to cases with strictly exogenous variables. Consider additional regressors $m_{i t}$ in model (1):

$$
\begin{aligned}
y_{i t}= & \mu_{i}+\left(\alpha_{1} y_{i t-1}+m_{i t}^{\prime} \beta_{1}\right) I\left(q_{i t} \leq \gamma\right) \\
& +\left(\alpha_{2} y_{i t-1}+m_{i t}^{\prime} \beta_{2}\right) I\left(q_{i t}>\gamma\right)+\varepsilon_{i t}
\end{aligned}
$$


TABLE 1: Quantiles of distribution, $\theta=0.1$.

\begin{tabular}{|c|c|c|c|c|c|c|c|c|c|}
\hline \multirow{2}{*}{ Quantiles } & \multicolumn{3}{|c|}{$T=10$} & \multicolumn{3}{|c|}{$T=15$} & \multicolumn{3}{|c|}{$T=20$} \\
\hline & 0.05 & 0.5 & 0.95 & 0.05 & 0.5 & 0.95 & 0.05 & 0.5 & 0.95 \\
\hline \multicolumn{10}{|l|}{$\gamma=2$} \\
\hline$N=100$ & 0.10 & 2.09 & 4.12 & 0.91 & 2.05 & 3.17 & 1.39 & 2.00 & 2.34 \\
\hline$N=200$ & 0.89 & 2.00 & 3.40 & 1.16 & 2.00 & 2.84 & 1.60 & 2.00 & 2.33 \\
\hline$N=300$ & -0.22 & 1.88 & 3.26 & 1.54 & 2.00 & 2.58 & 1.72 & 2.00 & 2.36 \\
\hline \multicolumn{10}{|l|}{$\alpha_{1}=0.5$} \\
\hline$N=100$ & 0.25 & 0.44 & 0.61 & 0.37 & 0.46 & 0.55 & 0.38 & 0.46 & 0.52 \\
\hline$N=200$ & 0.33 & 0.45 & 0.57 & 0.40 & 0.48 & 0.54 & 0.43 & 0.48 & 0.53 \\
\hline$N=300$ & 0.19 & 0.46 & 0.56 & 0.43 & 0.49 & 0.54 & 0.45 & 0.49 & 0.53 \\
\hline \multicolumn{10}{|l|}{$\alpha_{2}=0.6$} \\
\hline$N=100$ & 0.37 & 0.55 & 0.84 & 0.47 & 0.56 & 0.65 & 0.50 & 0.57 & 0.63 \\
\hline$N=200$ & 0.46 & 0.57 & 0.70 & 0.50 & 0.57 & 0.64 & 0.53 & 0.58 & 0.63 \\
\hline$N=300$ & 0.45 & 0.56 & 0.66 & 0.53 & 0.59 & 0.64 & 0.54 & 0.58 & 0.63 \\
\hline
\end{tabular}

TABLE 2: Quantiles of distribution, $\theta=0.3$.

\begin{tabular}{|c|c|c|c|c|c|c|c|c|c|}
\hline \multirow{2}{*}{ Quantiles } & \multicolumn{3}{|c|}{$T=10$} & \multicolumn{3}{|c|}{$T=15$} & \multicolumn{3}{|c|}{$T=20$} \\
\hline & 0.05 & 0.5 & 0.95 & 0.05 & 0.5 & 0.95 & 0.05 & 0.5 & 0.95 \\
\hline \multicolumn{10}{|l|}{$\gamma=2$} \\
\hline$N=100$ & 1.84 & 2.02 & 2.17 & 1.90 & 2.00 & 2.02 & 1.98 & 1.99 & 2.00 \\
\hline$N=200$ & 1.93 & 2.00 & 2.06 & 1.94 & 2.00 & 2.03 & 1.98 & 2.00 & 2.02 \\
\hline$N=300$ & 1.93 & 2.01 & 2.04 & 1.96 & 2.00 & 2.04 & 2.00 & 2.00 & 2.02 \\
\hline \multicolumn{10}{|l|}{$\alpha_{1}=0.5$} \\
\hline$N=100$ & 0.26 & 0.42 & 0.57 & 0.37 & 0.46 & 0.54 & 0.40 & 0.47 & 0.53 \\
\hline$N=200$ & 0.32 & 0.45 & 0.58 & 0.40 & 0.47 & 0.54 & 0.43 & 0.48 & 0.53 \\
\hline$N=300$ & 0.37 & 0.47 & 0.58 & 0.42 & 0.48 & 0.54 & 0.44 & 0.49 & 0.53 \\
\hline \multicolumn{10}{|l|}{$\alpha_{2}=0.8$} \\
\hline$N=100$ & 0.54 & 0.71 & 0.86 & 0.65 & 0.74 & 0.83 & 0.70 & 0.76 & 0.82 \\
\hline$N=200$ & 0.62 & 0.74 & 0.85 & 0.70 & 0.77 & 0.83 & 0.73 & 0.78 & 0.83 \\
\hline$N=300$ & 0.64 & 0.76 & 0.87 & 0.70 & 0.77 & 0.84 & 0.73 & 0.79 & 0.84 \\
\hline
\end{tabular}

for $i=1, \ldots, N ; t=1, \ldots, T$. Since $m_{i t}$ are strictly exogenous, they are valid instruments for the first differenced form of (22). Therefore, $\left(m_{i 2}^{\prime}, \ldots, m_{i T}^{\prime}\right)$ should be added to each diagonal element of $Z_{i}$ in (9). Hence, the matrix of instruments is

$$
\begin{gathered}
Z_{i} \\
=\left(\begin{array}{cccc}
x_{i 1}, m_{i 2}^{\prime} & 0 & \cdots & 0 \\
0 & x_{i, 1}, x_{i, 2}, m_{i 3}^{\prime} & \cdots & 0 \\
0 & 0 & \cdots & 0 \\
\vdots & \vdots & \ddots & \vdots \\
0 & 0 & \cdots & x_{i, 1}, x_{i, 2}, \ldots x_{i, T-1}, m_{i T}^{\prime}
\end{array}\right)
\end{gathered}
$$

then the estimators of $\gamma$ and slope coefficients $\left(\alpha^{\prime}, \beta^{\prime}\right)^{\prime}$ can be obtained accordingly as in (16) and (17).

\section{Monte Carlo Experiments}

In this section Monte Carlo experiments are implemented to examine the finite sample performance of our estimator. For this purpose we consider the following design.

4.1. Simulation Design. The data generating process (DGP) is given by

$$
y_{i t}=\mu_{i}+\alpha_{1} y_{i t-1} I\left(q_{i t} \leq \gamma\right)+\alpha_{2} y_{i t-1} I\left(q_{i t}>\gamma\right)+\varepsilon_{i t}
$$

for $i=1, \ldots, N$ and $t=1, \ldots, T$, where $\mu_{i} \sim$ i.i.d. $N(0,1)$, $q_{i t} \sim$ i.i.d. $N(2,1), \varepsilon_{i t} \sim$ i.i.d. $N(0,1)$, and $\mu_{i}, q_{i t}, \varepsilon_{i t}$ are mutually independent random variables. Let $y_{i 0}=0, \gamma=2$, $\alpha_{1}=0.5$, and $\theta=\alpha_{2}-\alpha_{1}=\{0.1,0.3\}$, and $N$ varies among $\{100,200,300\}$ and $T$ varies among $\{10,15,20\}$. All results are based on 1,000 replications.

The computation of the threshold $\gamma$ involves the minimization problem in (16), which can be reduced to searching for the values of $\gamma$ that minimizes the sum of squared errors among all distinct values of $q_{i t}$ in the sample. Obviously, there are at most NT distinct values of $q_{i t}$, and the minimum value of NT considered in the simulation is 1000. Thus, 


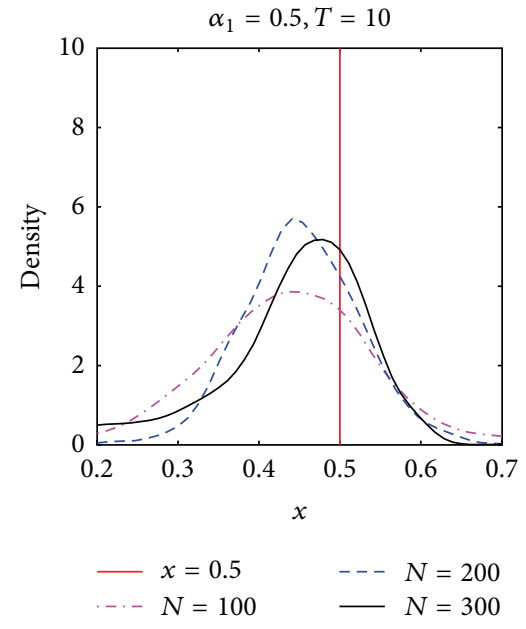

(a)

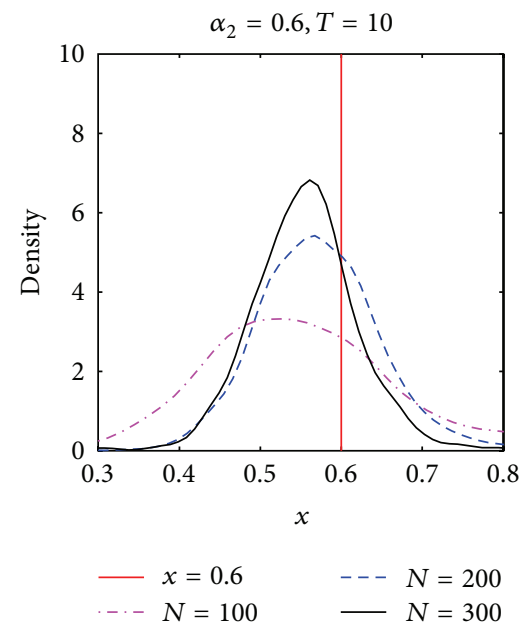

(d)

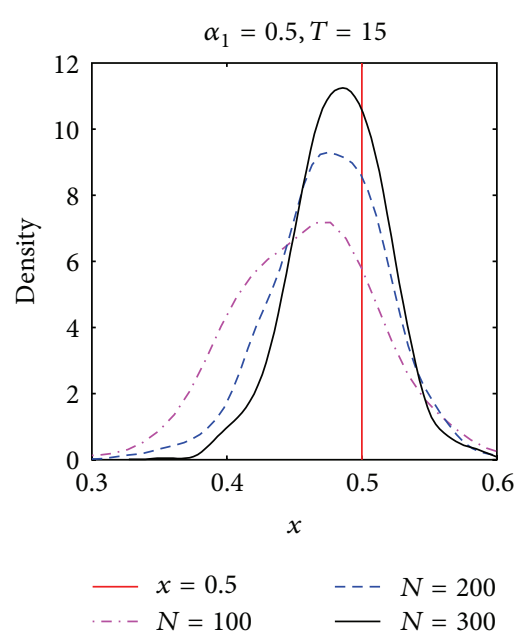

(b)

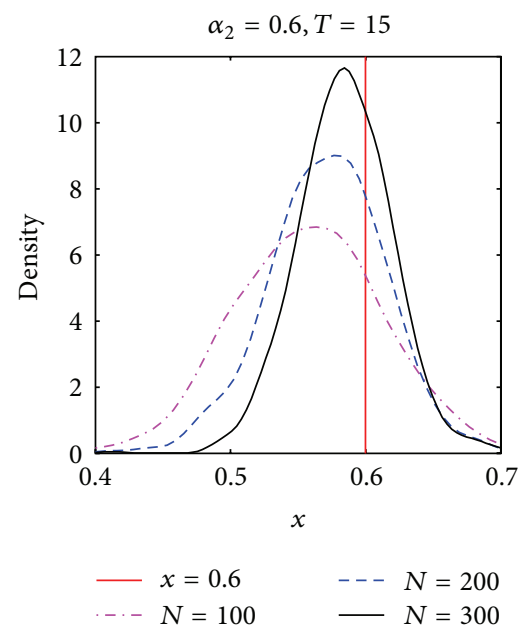

(e)

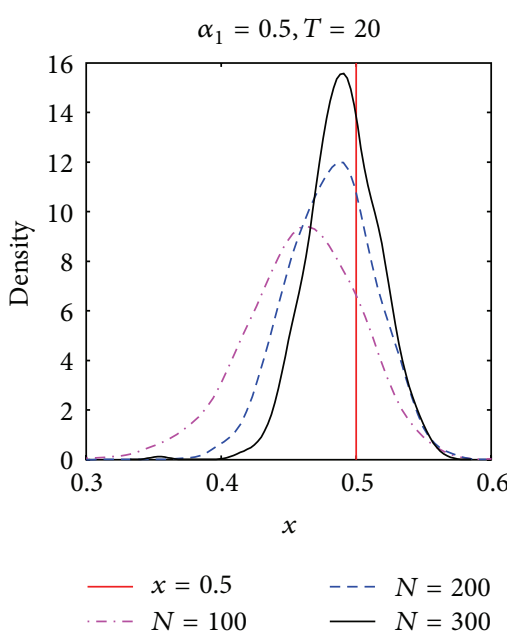

(c)

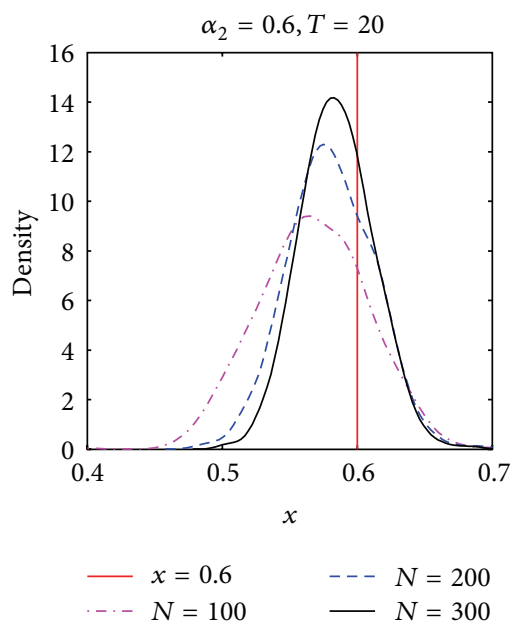

(f)

FIGURE 1: Density distribution of slope parameters (small threshold).

the searching could take a fair amount of time when the number of possible values is large. To reduce the computation load, we employ the method proposed by Hansen [17]. Specifically, instead of searching over all values of $q_{i t}$, we limit it to some specific quantiles $\{0.01,0.0125,0.015, \ldots, 0.99\}$, which contain only 393 different values. However, this approach may not be as appealing as searching over all possible values of $\gamma$ when the number of distinct value of $q_{i t}$ is small.

4.2. Simulation Results. Tables 1 and 2 represent the 5\%, 50\%, and $95 \%$ quantiles of the simulation distribution of $\widehat{\gamma}, \widehat{\alpha}_{1}$, and $\widehat{\alpha}_{2}$ for $T$ varying among 10,15 , and 20 and $N$ varying among 100,200 , and 300 .

Table 1 reports the results of $\theta=0.1$, corresponding to the case when threshold is small. The estimates of the threshold $\gamma$ perform fairly well for all cases considered, since the medians of $\hat{\gamma}$ are around the true value $\gamma=2$. As $T$ increases, the distribution of $\hat{\gamma}$ is becoming more and more concentrated around the true value. For example, when $N=$ 100 and $T=10$, the length of the quantile range between 0.05 and 0.95 is 4.02 , while, when $T=20$, the length decreases to 0.95 . The distribution of the slope coefficient estimator $\widehat{\alpha}_{1}$ exhibits a little downward bias as it has been shown in some of the existing Monte Carlo studies for dynamic panel data models. For $N=100$ and $T=10$, the median bias of $\widehat{\alpha}_{1}$ is 0.06 , but this bias is reduced as $N$ and/or $T$ increases; for example, this bias is only 0.01 for $N=300$ and $T=20$. Similarly, the length of the quantile range between 0.05 and 0.95 for $\widehat{\alpha}_{1}$ is getting smaller as $T$ increases, which means that the performance improves. The quantiles of the distribution of $\widehat{\alpha}_{2}$ also performs well in all cases, although it is relatively weak in cases with small $T$ and small $N$.

Table 2 presents the results for the case when threshold is big; that is, $\theta=0.3$. Compared to the small threshold case, the performance of the distribution of $\hat{\gamma}$ is improved. The median bias of $\hat{\gamma}$ is zero for almost all cases, and the length of the quantile range between 0.05 and 0.95 is getting smaller as the threshold effect increases. Meanwhile, Table 2 reports similar results as Table 1 for the parameters of $\widehat{\alpha}_{1}$ and $\widehat{\alpha}_{2}$. In Table 2 , they also perform fairly well in the big threshold case. 


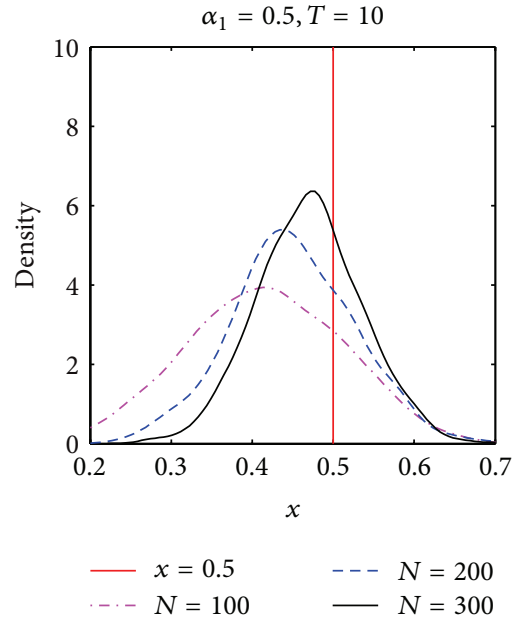

(a)

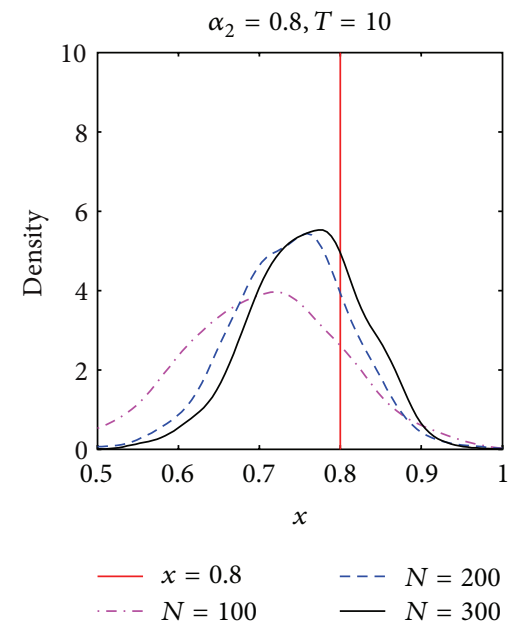

(d)

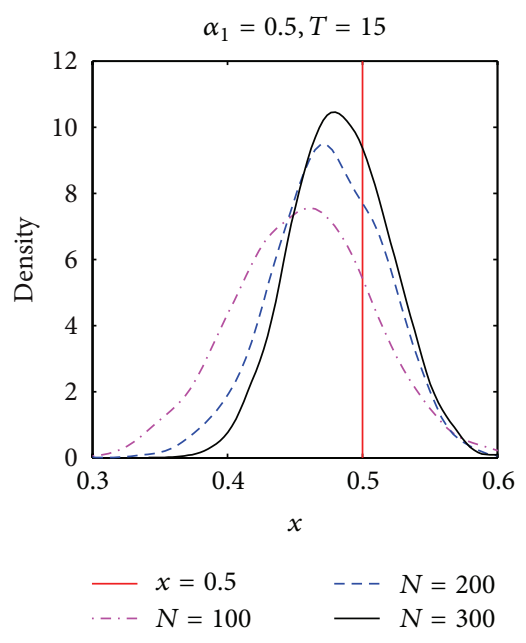

(b)

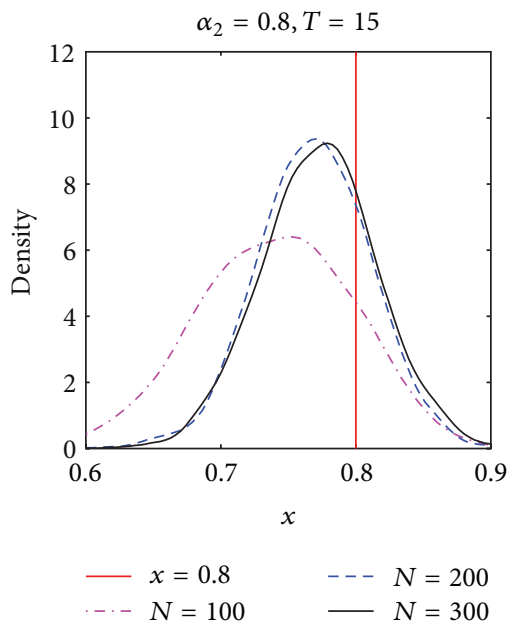

(e)

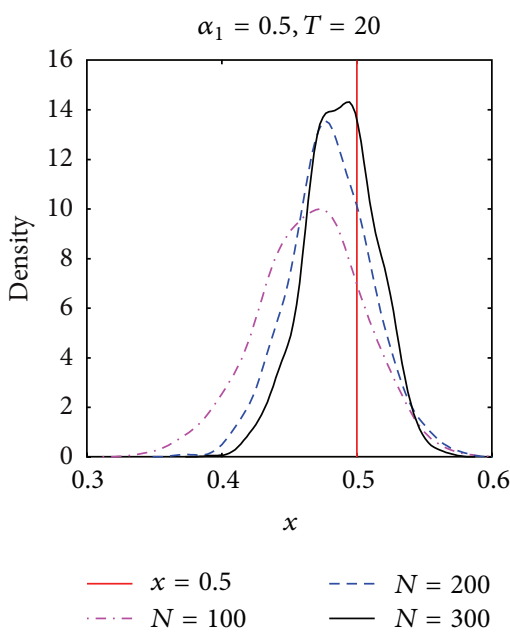

(c)

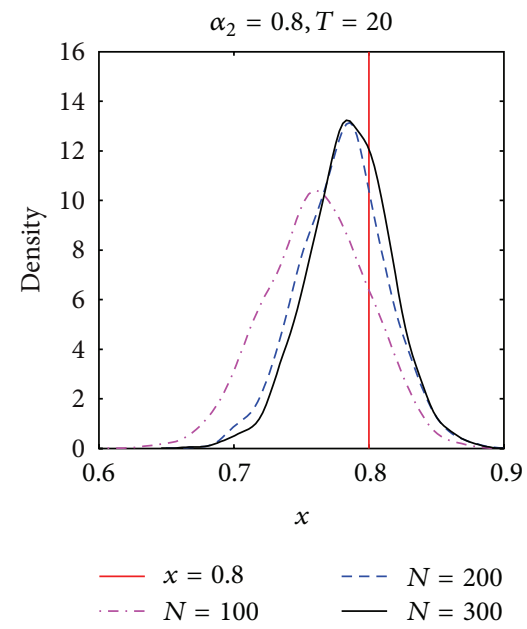

(f)

FIGURE 2: Density distribution of slope parameters (big threshold).

Figure 1 displays kernel estimates of the distribution of the slope parameters $\widehat{\alpha}_{1}$ and $\widehat{\alpha}_{2}$ based on 1,000 replications with $N=100,200,300, T=10,15,20$, and small threshold $(\theta=0.1)$. The estimates are slightly biased downwards when $T$ is small or $N$ is small. This bias is common in dynamic panel data model as mentioned earlier. One could also use some bias-corrected methods to improve the finite sample properties of the estimators, which is beyond the scope of this paper. The estimates are gradually centered around the true values as $N$ and/or $T$ increases, which is consistent with the above analyses and confirms the validity of our proposed estimation procedure again.

Figure 2 shows the distribution of the same parameters as Figure 1 and based on the same number of replications and sample size but with bigger threshold $(\theta=0.3)$. In this case the same conclusion can be found as in Figure 1. In particular, the performance of the estimators in this case is better than that in the smaller threshold for all cases.

\section{Conclusion}

This paper extends the estimation of threshold models in nondynamic panels to dynamic panels and presents practical estimation methods for these econometric models with individual-specific effects and threshold effects. The foremost feature of these models is that they allow the econometrician to consider the dynamic and threshold relationships in economic system simultaneously. As mentioned in the introduction, many applications may have such relationships. Using the first-difference to eliminate the individual-specific effects, we prove that the orthogonality conditions proposed by Arellano and Bond [10] for nonthreshold models are also valid in our models. Then, we estimate the threshold and slope parameters by GMM. Monte Carlo simulations reveal that our method has very good finite sample performance.

There are several possible extensions to this work. The asymptotic properties of the threshold parameter would be 
an interesting topic. Also, testing for one or multiple thresholds is also worth studying, which is saved for future research.

\section{Conflict of Interests}

The authors declare that there is no conflict of interests regarding the publication of this paper.

\section{Acknowledgments}

The authors thank the editor and three anonymous referees for many constructive and helpful comments. This work was partially supported by the National Natural Science Foundation of China (Grant nos. 71301160 and 71301173), China Postdoctoral Science Foundation funded project (Grant nos. 2012M520419, 2012M520420, and 2013T60186), Beijing Planning Office of Philosophy and Social Science (13JGB018), and Program for Innovation Research in Central University of Finance and Economics.

\section{References}

[1] C. X. Huang, C. L. Peng, X. H. Chen, and F. H. Wen, "Dynamics analysis of a class of delayed economic model," Abstract and Applied Analysis, vol. 2013, Article ID 962738, 12 pages, 2013.

[2] F. Wen and Z. Dai, "Modified Yabe-Takano nonlinear conjugate gradient method," Pacific Journal of Optimization, vol. 8, no. 2, pp. 347-360, 2012.

[3] O. Galor and D. N. Weil, "Population, technology, and growth: from malthusian stagnation to the demographic transition and beyond," The American Economic Review, vol. 90, no. 4, pp. 806$828,2000$.

[4] A. Mas-colell and A. Razin, "A model of intersectioral migration and growth," Oxford Economic Papers, vol. 25, no. 1, pp. 72-79, 1973.

[5] P. F. Peretto, "Industrial development, technological change, and long-run growth," Journal of Development Economics, vol. 59, no. 2, pp. 389-417, 1999.

[6] T. W. Anderson and C. Hsiao, "Estimation of dynamic models with error components," Journal of the American Statistical Association, vol. 76, no. 375, pp. 598-606, 1981.

[7] A. Ciarreta and A. Zarraga, "Economic growth-electricity consumption causality in 12 European countries: a dynamic panel data approach," Energy Policy, vol. 38, no. 7, pp. 37903796, 2010.

[8] T. S. Eicher and T. Schreiber, "Structural policies and growth: time series evidence from a natural experiment," Journal of Development Economics, vol. 91, no. 1, pp. 169-179, 2010.

[9] B.-N. Huang, M. J. Hwang, and C. W. Yang, "Causal relationship between energy consumption and GDP growth revisited: a dynamic panel data approach," Ecological Economics, vol. 67, no. 1, pp. 41-54, 2008.

[10] M. Arellano and S. Bond, "Some tests of specification for panel data: Monte Carlo evidence and an application to employment equations," The Review of Economic Studies, vol. 58, pp. 277-297, 1991.

[11] M. Arellano and O. Bover, "Another look at the instrumental variable estimation of error-components models," Journal of Econometrics, vol. 68, no. 1, pp. 29-51, 1995.
[12] R. Blundell and S. Bond, "Initial conditions and moment restrictions in dynamic panel data models," Journal of Econometrics, vol. 87, no. 1, pp. 115-143, 1998.

[13] B. H. Baltagi, Econometric Analysis of Panel Data, John Wiley \& Sons, Chichester, UK, 2008.

[14] C. Han and P. C. B. Phillips, "GMM estimation for dynamic panels with fixed effects and strong instruments at unity," Econometric Theory, vol. 26, no. 1, pp. 119-151, 2010.

[15] B. E. Hansen, "Sample splitting and threshold estimation," Econometrica, vol. 68, no. 3, pp. 575-603, 2000.

[16] M. Caner and B. E. Hansen, "Instrumental variable estimation of a threshold model," Econometric Theory, vol. 20, no. 5, pp. 813-843, 2004

[17] B. E. Hansen, "Threshold effects in non-dynamic panels: estimation, testing, and inference," Journal of Econometrics, vol. 93, no. 2, pp. 345-368, 1999.

[18] J. Cheng, C. Lin, and C. Wang, "Estimation of growth convergence using common correlated effects approaches," Working Paper, 2009.

[19] T. T. L. Chong, Q. He, and M. J. Hinich, "The nonlinear dynamics of foreign reserves and currency crises," Studies in Nonlinear Dynamics \& Econometrics, vol. 12, no. 4, article 2, 2008.

[20] T.-W. Ho, "Income thresholds and growth convergence: a panel data approach," Manchester School, vol. 74, no. 2, pp. 170-189, 2006.

[21] S. Kremer, A. Bick, and D. Nautz, "Inflation and growth: new evidence from a dynamic panel threshold analysis," Empirical Economics, vol. 44, pp. 861-878, 2013.

[22] V. A. Dang, M. Kim, and Y. Shin, "Asymmetric capital structure adjustments: new evidence from dynamic panel threshold models," Journal of Empirical Finance, vol. 19, no. 4, pp. 465482, 2012.

[23] K. S. Chan, "Consistency and limiting distribution of the least squares estimator of a threshold autoregressive model," The Annals of Statistics, vol. 21, no. 1, pp. 520-533, 1993.

[24] L. P. Hansen, "Large sample properties of generalized method of moments estimators," Econometrica, vol. 50, no. 4, pp. 10291054, 1982.

[25] F. Windmeijer, "A finite sample correction for the variance of linear efficient two-step GMM estimators," Journal of Econometrics, vol. 126, no. 1, pp. 25-51, 2005. 


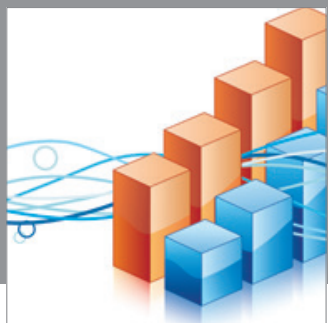

Advances in

Operations Research

mansans

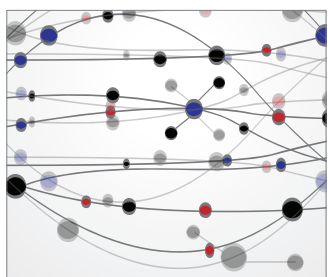

The Scientific World Journal
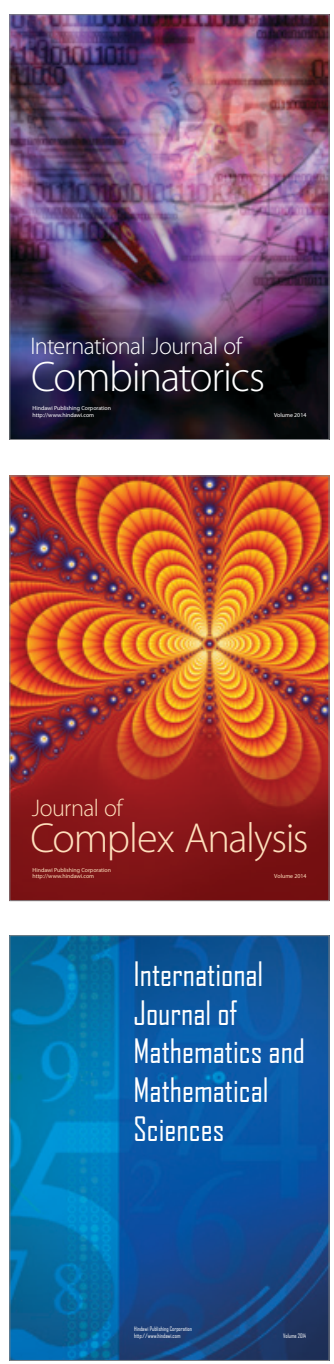
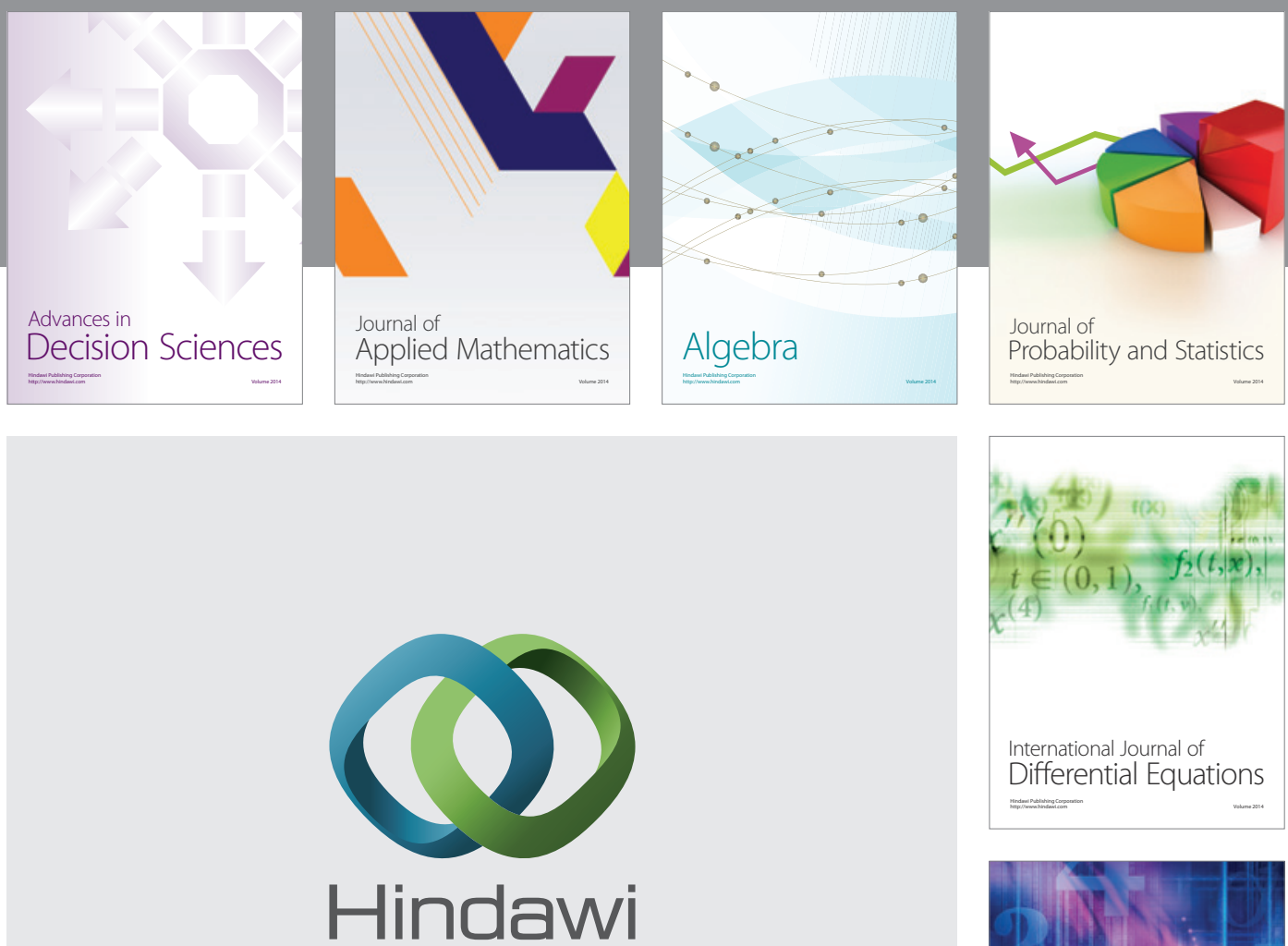

Submit your manuscripts at http://www.hindawi.com
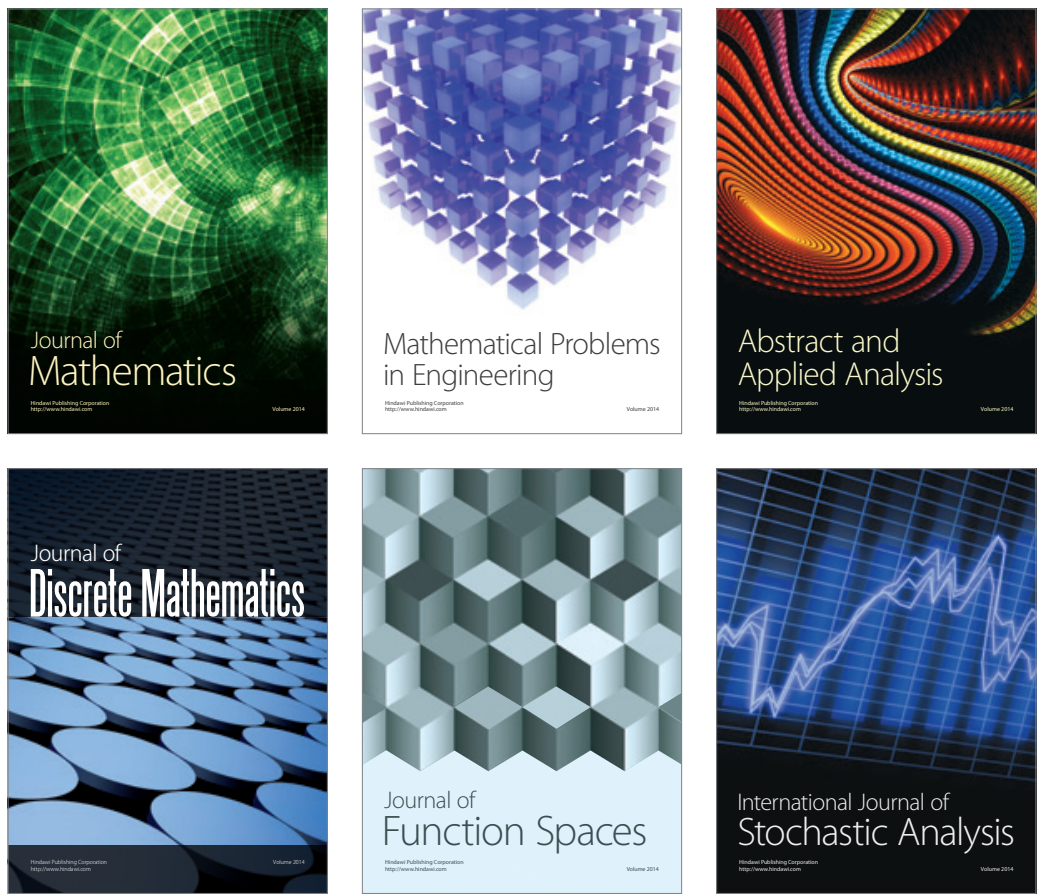

Journal of

Function Spaces

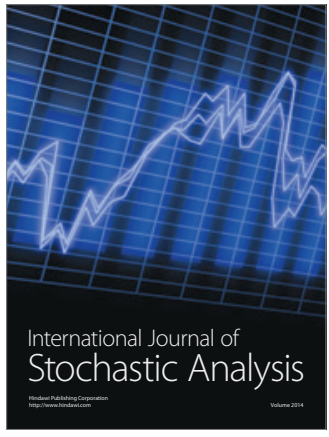

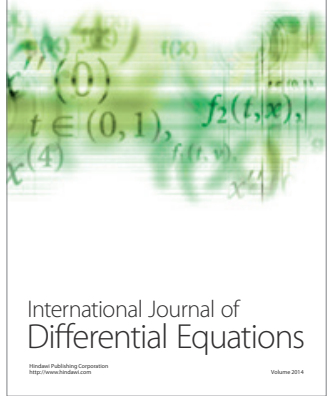
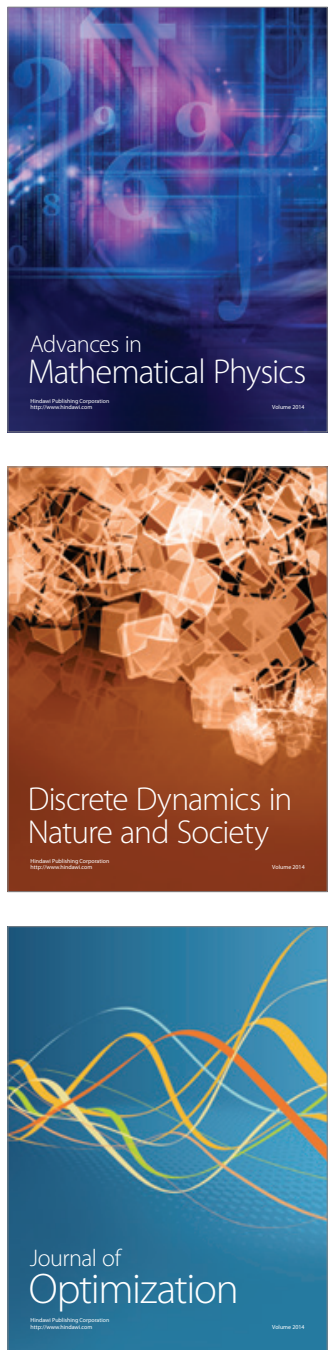